\title{
Oscillatory Changes in Structural Characteristics in Multicomponent Ceramics, YBCO
}

\author{
Boris Kodess ${ }^{1,2, a^{*}}$ and Pavel Kodess ${ }^{2, b}$ \\ ${ }^{1}$ VNIIMS, 46 Ozernaya Str, 119361, Moscow, Russia \\ ${ }^{2}$ ICS\&E, 919 Telluride St., 80011 Aurora, CO, USA \\ akodess@mail.ru, bicse96co@gmail.com
}

\begin{abstract}
Keywords: Multicomponent Ceramics, Yttrium Barium Cuprate, YBCO, X-Ray Irradiation, Long-Time Series, Active Oxygen, Breathing Modes of Behavior, Compression and Expansion Waves, Macrostresses, Complexity, Nonlinear Dynamics, Self-Organization Process
\end{abstract}

\begin{abstract}
The structural characteristics of samples of a four-component superconducting material (YBCO) after exposure to X-ray irradiation during a long time are investigated. The effect of Xray beam processing on angular positions (corresponding parameters of the crystal lattice) and the width of Bragg reflections is established. The phenomenon of oscillatory behavior in the unit cell dimension with long-time irradiation is found. The analysis of the profiles of reflection also demonstrates the presence of reversible changes phase composition with the exposure time. The observed phenomena reflect the presence of a nontrivial and specific process of compression and expansion of the unit cell due to the accumulation and then disengagement outside of ionized oxygen, which is formed under such irradiation exposure on the surface of the samples.
\end{abstract}

\section{Introduction}

Irradiation processing is widely used for alloying. This type of impact on materials needs to be considered in some applications. For example, after the exposure to neutron irradiation of substances in which there are covalent chemical bonds, there happen either a change in the unit cell dimension [1-2], or even transformation of part of the volume into another element.

There is a class of substances in which, along with rigid covalent and ionic types of chemical bonds, there also exist weaker chemical bonds. We observed the impact of soft X-ray irradiation on the structural characteristics of the known material DKDP (potassium dihydrogen phosphate) for the formation of biocrystals and the used in laser devices [3]. We also [4-5] studied the effect of maturing in the long-term X-ray exposure on the well-known superconducting four-component ceramic material YBCO (YBa2Cu3O7-x, yttrium barium cuprate).

The distinctive feature of this substance is that oxygen, when chemically bound with these metals - Y, Ba, $\mathrm{Cu}$ - exist in three different charge states. These include oxygen bound with copper atoms in unit cell and which is in a nearly neutral state. Also, in [4] the changes were observed after a long maturing of the samples. Note that breathing modes of behavior of atoms are inherent, and most likely observed, for large macromolecules in bioactive substances. This phenomenon is unique for inorganic materials.

In this article, we present the results from a new time-series experiment for YBCO samples, when measurements were carried out continuously, and discuss the possible mechanism of the observed oscillatory processes in it. 


\section{Samples and procedures}

YBCO samples were taken from a batch prepared as certified standard materials of composition and diffraction properties (GSO PRF-23) and had the composition YBa2Cu3O6.93. The superconducting transition temperature, $\mathrm{Tc}=91,5 \mathrm{~K}$, has been maintained for over 30 years since the creation of this batch. Also during this period, the characteristics of the full diffraction pattern were repeatedly measured. The calculations of the structural characteristics, carried out using the Rietveld method, confirmed the invariability of the composition of these samples.

More detailed continuous measurements of the characteristics of the diffraction pattern during the X-ray exposure on the surface of the samples were carried out on the DRON-4 diffractometer (CuKa-radiation) and followed by additional measurements having much softer VKa-radiation and $\mathrm{CrKa}$-radiation and then much harder MoKa-radiation. The oscillating changes in the angular positions and the shape of the profiles were revealed; however, after some time in the course of the measurements, the angular position of the reflections, corresponding to the change in the crystal lattice parameter, began to return to the initial state. A typical picture is shown in Fig. 1.

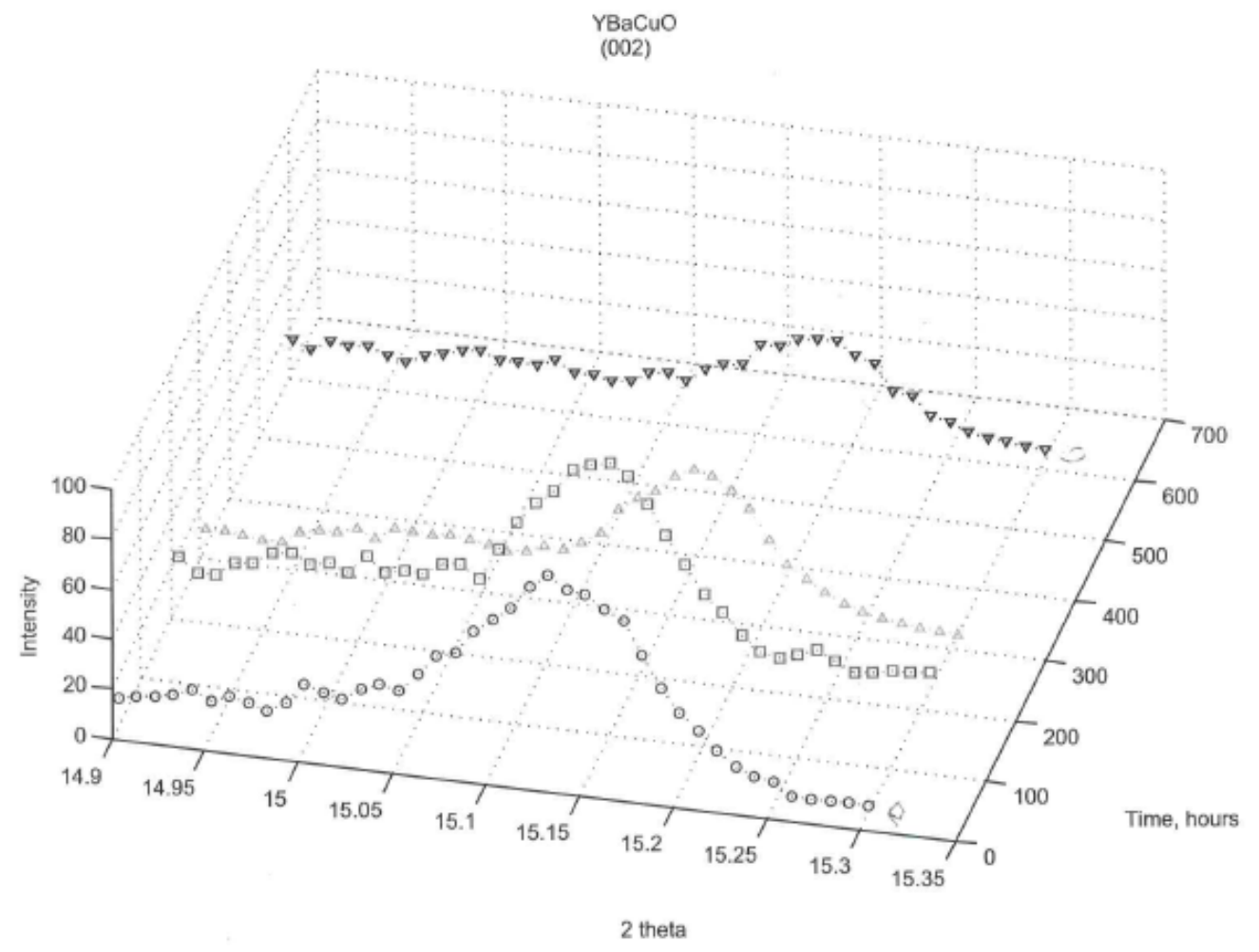

Fig. 1. An example of changes in a diffraction reflection with Miller indices (002) at different time intervals of observation.

To analyze the profiles the «Profan» program was used. The results showed that after the exposure to the X-ray beam, they can be described as a set of several profiles and in terms of the appearance of reflections corresponding to compositions with higher oxygen content. For softer VKa- and CrKa-radiations, the changes started faster and were more pronounced, and the opposite picture was observed under MoKa-radiation.

\section{Discussion}

The structural characteristics and properties of ceramic and single-crystal YBCO samples with different oxygen contents were studied in great detail by various methods [6]. When studying the 
effect of laser irradiation of YBCO films, a change in the relief was found due to growth defects which are the results of relaxations of the accumulating strains [7].

The investigation of the photoresponse of YBCO films [8] showed that the relaxation of excited quasiparticles can occur owing to localized states near the Fermi level. The mechanism of similar photoinduced reactions based on the results of X-ray crystallographic and spectroscopic data was considered for proteins [9] and other substances [15].

The X-rays with different wavelengths, applied by us, gave the penetration into the analyzed sample volume of different depths. The obtained results indicate that the observed phenomenon occurs mostly in the near-surface regions of the samples. The appearance of additional reflections, reflecting higher oxygen content, means the oxygen penetration into the layers of surface. This process is possible because when exposed to X-rays, the air is noticeably ionized, and there is a part of more active oxygen (ozone) in it. Such oxygen saturates vacancies in the basal plane of the unit cell. In that part of the vacancies some also contained oxygen with the charge of such atom being close to neutral. An anomalously high diffusion rate was then observed several times for this compound of this atomic plane.

An increase in the oxygen content in a unit cell leads to a noticeable compression waves in this cell and the appearance of micro- and macrostresses in the sample. Indeed, we observed spontaneous bending of plates made of this material after a long maturing, which may be associated with relaxation of macrostresses due to the arising of compression waves. The presence of the compressed powder in quartz cuvettes for X-ray diffraction also led to cracking of the cuvettes holding this powder. This observation reflects a very high level of such macrostresses, capable of destroying a sufficiently strong material.

The YBCO unit cell appeared because of the replacement of lanthanum and strontium in the LaSrCuO4-x compound for yttrium and barium, which led to a strong chemical contraction of this cell, along with an increase in Tc from 39 to $92 \mathrm{~K}$. We considered a similar chemical contraction effect for another superconducting compound V3Si [10]. In this case, due to the chemical contraction of $\mathrm{V}$ atoms by $\mathrm{Si}$ atoms with covalent bonds, the value of $\mathrm{Tc}$ also increased almost three-fold.

Moreover, we observed the phenomenon of strong swelling of V3Si, milled to a fraction of 20 microns and pressed in cuvettes when the maturing process took place. In some cases, when the milled particles decreased to 3-5 microns, the cracking of quartz cuvettes was also observed, similar to that observed for YBCO.

The nonlinearity of the response of a multicomponent system to external influences at the electronic and atomic level of structure was inherited during the transition to a larger scale of the structure [11-12] and vice versa. For several samples this manifested itself in the appearance of chaotic phenomena and avalanches when exposed to a strong magnetic field [13-14] of near the superconducting state.

It should be noted that the process of self-organization in highly nonlinear systems can be associated with bifurcations and different trajectories of the dynamic process. Therefore, the longer observation we made, we were able to see different sequences of changes in the diffraction pattern and the contribution of the emerging regions with different oxygen contents. However, despite the different levels of oscillations of the structural characteristics, they all returned to their initial state.

The oscillatory phenomena are well studied in the reaction-diffusion systems [15]. In [16], on the basis of synergetic approaches, as a theory of self-organizing systems, it is shown that the controlling of the structure and properties is related to establishing conditions of spontaneously 
released elastic energy as the system loses its space-time symmetry being an independent source of energy.

At the atomic level, however, this observation is considered to be rather unique. Therefore, the YBCO samples were also provided to the Division of Material Reliability, NIST, USA, where they were analyzed on their X-ray diffractometer. The presence of oscillatory changes in the characteristics of the diffraction pattern was confirmed, which shows the reproducibility of the observed phenomenon.

Summary

The emerging of spatio-temporal patterns demonstrates the process of self-organization in this ceramics at the atomic and microscopic levels as well as the presence of peculiar 'breathing' modes of behavior of this multicomponent material. At the same time, the added complexity of the structure of the material by several components with variable concentration leads to the dynamics of processes, which has a more pronounced nonlinear character, and a variety of the observed spatio-temporal patterns.

Acknowledgments

The authors are grateful to Prof. D. Balzar (NIST, Denver University, CO, USA) for reproducing part of a time-series of YBCO samples, and to I.L. Kommel and A.A. Ignatov for their help in carrying out very long-time series of measurements, and G.V. Bitukova, I. P, Jouravlev and G.V. Guschin for their help in processing a large amount of accumulated data.

\section{References}

[1] L.A. Vasilyeva, M.I. Boychuk, S.A. Mikaeva, Radiation resistance of quartz generators. Book of reference. Engineering Journal with supplement, 8 (2020) 23-29. https://doi.org/10.14489/hb.2020.08.pp.023-029

[2] B.N. Kodess, V.A. Sarin. A Neutron diffractometer for determining the structural characteristics of single crystals, Measurement Techniques, 57(11), (2015) 1299-1303. https://doi.org/10.1007/s11018-015-0624-3

[3] N.S. Kolesova, G.P. Revkevich, B.N. Kodess, A.A. Katznelson, Effect of soft X-ray radiation on structural characteristics of $\mathrm{KD}_{2} \mathrm{P0}_{4}$. Moscow University, Physics Bulletin, Sect. 3, Physics and Astronomy 50(4), (1995) 107

[4] B.N. Kodess, M.T. Medetbekov, The Processes Self-Organization of Structural Parameters Investigation in HTSP materials. VNIIMS, 25 December 1995, Moscow, Depozit VINITI, 04G113Dep., N 2075-B96 (rus); (1996), 48p.: B.N. Kodess, Self-Oscillations in a crystalline substance. Nature, 04/09/1995, K096, (1995) 1-6

[5] V.M. Avduchina, N.S. Kolesova, B.N. Kodess, A.A. Katznelson, G.P., Revkevich, S.M. Zivotov, Structure Changes in Crystals with Various Nonstoichiometries under Long Aging and Exciting Influence. Surface Investigation (Physics, Chemistry, Mechanics), 12 (1997) 695-709

[6] B.N. Kodess, L.A. Butman, M.A. Poray-Koshits, On Yttrium Metalloxide Stability. (Engl. Transl.) Sov. Physics DOKLADY, 35(1), (1990) 60-62

[7] V.D. Okunev, Z.A. Samoilenko, S.J. Lewandowski, T.A. D’yachenko, V.A. Isaev, E.I. Pushenko, V.N. Varyukhin, P. Gierlowski, S. Barbanera, Effect of laser irradiation on the structure and properties of dielectric column clusters in the $\mathrm{YBaCuO}$ superconducting films. Technical Physics, 58(2), (2013) 231-238. https://doi.org/10.1134/S1063784213020163 
[8] J. Demsar, M. Zavrtanik, B. Podobnik, V. I. Dediu \& D. Mihailovic, Photoresponse of Y-Ba$\mathrm{Cu}-\mathrm{O}$ thin rims on the Ms time scale-evidence for localized states near $\mathrm{E}_{\mathrm{F}}$. Journal of Superconductivity, 10(4), (1997) 455-458. https://doi.org/10.1007/BF02765737

[9] M.G. Khrenova' A.V. Nemukhin, B.L. Grigorenko, A.I. Krylov, T.M. Domratcheva, Quantum chemistry calculations provide support to the mechanism of the light-induced structural changes in the flavin-binding photoreceptor proteins. J. Chem. Theory Comput. 6(8), (2010) 293-2302. https://doi.org/10.1021/ct100179p

[10] B.N. Kodess, F.A. Sidorenko, Anisotropy of Magnetic Susceptibility of the V3Si Silicide, Physics of Metals and Metallography, 122(4), (2021) 345-350.

https://doi.org/10.1134/S0031918X21040037

[11] B.N. Kodess, Multi-factor analysis in metrology of materials and its correlation with the System of Units of measurements, Legal and applied metrology, (1), (2021) 46-51

[12] B.N. Kodess, P.B. Kodess, Reference data and multi-factorial methods for development of modern materials, Material Science Forum, K0646, (2022) 1-10 (to published)

[13] V.V. Chabanenko, B.N. Kodess, N.V. Kuzovoi, H. Szymczak, Fine Structure of Thermal Runaway Process in the V3Si Single-crystal Superconductor as a Result of Pinning Center Response, Physics Procedia, 36 (2012) 634-637. https://doi.org/10.1016/j.phpro.2012.06.181

[14] A. Nabialek, V. Chabanenko, S. Vasiliev, G. Shushmakova, H. Szymczak, B. Kodess, Two components of the magnetostriction of the crystalline metallic V3Si superconductor, Journal of Applied Physics, 105(6), (2009) 063918-063918. https://doi.org/10.1063/1.3093696

[15] V.K. Vanag, Waves and patterns in reaction-diffusion systems. Belousov-Zhabotinsky reaction in water-in-oil microemulsions, Physics-Uspekhi 47(9), (2004) 923-941. https://doi.org/10.1070/PU2004v047n09ABEH001742

[16] V.S. Ivanova, A.A. Shanyavsky, Nanoscience as a theory of control over the structure and properties of nanosystems, Metallurgia mashinostroyeniya 6, (2008) 31-35 (rus) 